\title{
Research Article \\ Extended Mapping Method and Its Applications to Nonlinear Evolution Equations
}

\author{
J. F. Alzaidy \\ Mathematics Department, Faculty of Science, Taif University, Saudi Arabia \\ Correspondence should be addressed to J. F. Alzaidy, j-f-h-z@hotmail.com
}

Received 2 April 2012; Revised 15 July 2012; Accepted 31 July 2012

Academic Editor: Renat Zhdanov

Copyright (C 2012 J. F. Alzaidy. This is an open access article distributed under the Creative Commons Attribution License, which permits unrestricted use, distribution, and reproduction in any medium, provided the original work is properly cited.

We use extended mapping method and auxiliary equation method for finding new periodic wave solutions of nonlinear evolution equations in mathematical physics, and we obtain some new periodic wave solution for the Boussinesq system and the coupled KdV equations. This method is more powerful and will be used in further works to establish more entirely new solutions for other kinds of nonlinear partial differential equations arising in mathematical physics.

\section{Introduction}

The effort in finding exact solutions to nonlinear equations is important for the understanding of most nonlinear physical phenomena. For instance, the nonlinear wave phenomena observed in fluid dynamics, plasma, and optical fibers are often modeled by the bellshaped sech solutions and the kink-shaped tanh solutions. Many effective methods have been presented, such as inverse scattering transform method [1], Bäcklund transformation [2], Darboux transformation [3], Hirota bilinear method [4], variable separation approach [5], various tanh methods [6-9], homogeneous balance method [10], similarity reductions method [11, 12], ( $\left.G^{\prime} / G\right)$-expansion method [13], the reduction mKdV equation method [14], the trifunction method $[15,16]$, the projective Riccati equation method [17], the Weierstrass elliptic function method [18], the Sine-Cosine method $[19,20]$, the Jacobi elliptic function expansion [21, 22], the complex hyperbolic function method [23], the truncated Painlevé expansion [24], the F-expansion method [25], the rank analysis method [26], the ansatz method [27, 28], the exp-function expansion method [29], and the sub-ODE method [30].

The main objective of this paper is using the extended mapping method to construct the exact solutions for nonlinear evolution equations in the mathematical physics via the Boussinesq system and the coupled KdV equations. 


\section{Description of the Extended Mapping Method}

Suppose we have the following nonlinear PDE:

$$
F\left(u, u_{t}, u_{x}, u_{t t}, u_{x x}, u_{x t}, \ldots\right)=0 \text {, }
$$

where $u=u(x, t)$ is an unknown function, $F$ is a polynomial in $u=u(x, t)$ and its various partial derivatives in which the highest order derivatives and nonlinear terms are involved. In the following we give the main steps of a deformation method.

Step 1. The traveling wave variable

$$
u(x, t)=u(\xi), \quad \xi=k(x-\omega t),
$$

where $k$ and $\omega$ are the wave number and the wave speed, respectively. Under the transformation (2.2), (2.1) becomes an ordinary differential equation (ODE) as

$$
P\left(u, u^{\prime}, u^{\prime \prime}, u^{\prime \prime \prime}, \ldots\right)=0 .
$$

Step 2. If all the terms of (2.3) contain derivatives in $\zeta$, then by integrating this equation and taking the constant of integration to be zero, we obtain a simplified ODE.

Step 3. Suppose that the solution (2.3) has the following form:

$$
u(\xi)=a_{0}+\sum_{i=1}^{n}\left(a_{i} f^{i}(\xi)+b_{i} f^{-i}(\xi)\right)+\sum_{i=2}^{n} c_{i} f^{i-2}(\xi) f^{\prime}(\xi)+\sum_{i=-1}^{n} d_{i} f^{i}(\xi) f^{\prime}(\xi),
$$

where $a_{0}, a_{i}, b_{i}, c_{i}$, and $d_{i}$ are constants to be determined later, while $f(\xi)$ satisfies the nonlinear ODE:

$$
\left[f^{\prime}(\xi)\right]^{2}=p f^{4}(\xi)+q f^{2}(\xi)+r
$$

where $p, q$, and $r$ are constants.

Step 4 . The positive integer " $n$ " can be determined by considering the homogeneous balance between the highest derivative term and the nonlinear terms appearing in (2.3). Therefore, we can get the value of $n$ in (2.4).

Step 5. Substituting (2.4) into (2.3) with the condition (2.5), we obtain polynomial in $f^{i}(\xi)\left[f^{\prime}(\xi)\right]^{j},(i=\ldots,-2,-1,0,1,2, \ldots ; j=0,1)$. Setting each coefficient of this polynomial to be zero yields a set of algebraic equations for $a_{0}, a_{i}, b_{i}, c_{i}, d_{i}, \omega$, and $k$.

Step 6. Solving the algebraic equations by use of Maple or Mathematica, we have $a_{0}, a_{i}, b_{i}, c_{i}$, $d_{i}$, and $k$ expressed by $p, q, r$. 
Step 7. Since the general solutions of (2.5) have been well known for us (see Appendix A), then substituting the obtained coefficients and the general solution of (2.5) into (2.4), we have the travelling wave solutions of the nonlinear PDE (2.1).

\section{Applications of the Method}

In this section, we apply the extended mapping method to construct the exact solutions for the Boussinesq system and the coupled $\mathrm{KdV}$ equations, which are very important nonlinear evolution equations in mathematical physics and have been paid attention by many researchers.

Example 3.1 (the Boussinesq system). We start the Boussinesq system [32] in the following form:

$$
\begin{gathered}
v_{t}=\frac{1}{3} u_{x x x}+\frac{8}{3} u u_{x}, \\
u_{t}=v_{x} .
\end{gathered}
$$

The traveling wave variable (2.2) permits us converting (3.1) into the following ODE:

$$
\begin{gathered}
\omega v^{\prime}+\frac{1}{3} k^{2} u^{\prime \prime \prime}+\frac{8}{3} u u^{\prime}=0, \\
\omega u^{\prime}+v^{\prime}=0 .
\end{gathered}
$$

Integrating (3.2) with respect to $\xi$ once and taking the constant of integration to be zero, we obtain

$$
\begin{gathered}
\omega v+\frac{1}{3} k^{2} u^{\prime \prime}+\frac{4}{3} u^{2}=0 \\
\omega u+v=0
\end{gathered}
$$

Suppose that the solutions of (3.3) and (3.4) can be expressed by

$$
\begin{gathered}
u(\xi)=a_{0}+\sum_{i=1}^{n}\left(a_{i} f^{i}(\xi)+b_{i} f^{-i}(\xi)\right)+\sum_{i=2}^{n} c_{i} f^{i-2}(\xi) f^{\prime}(\xi)+\sum_{i=-1}^{-n} d_{i} f^{i}(\xi) f^{\prime}(\xi), \\
v(\xi)=A_{0}+\sum_{i=1}^{m}\left(A_{i} f^{i}(\xi)+B_{i} f^{-i}(\xi)\right)+\sum_{i=2}^{m} L_{i} f^{i-2}(\xi) f^{\prime}(\xi)+\sum_{i=-1}^{-m} H_{i} f^{i}(\xi) f^{\prime}(\xi),
\end{gathered}
$$

where $a_{0}, a_{i}, b_{i}, c_{i}, d_{i}, A_{i}, B_{i}, L_{i}$, and $H_{i}$ are constants to be determined later. 
Considering the homogeneous balance between the highest order derivative $u^{\prime \prime}$ and the nonlinear term $u^{2}$ in (3.3), the order of $u$ and $v$ in (3.4), then we can obtain $n=m=2$, hence the exact solutions of (3.5) can be rewritten as,

$$
\begin{gathered}
u(\xi)=a_{0}+a_{1} f(\xi)+b_{1} \frac{1}{f(\xi)}+a_{2} f^{2}(\xi)+b_{2} \frac{1}{f^{2}(\xi)}+c_{2} f^{\prime}(\xi)+d_{1} \frac{f^{\prime}(\xi)}{f(\xi)}+d_{2} \frac{f^{\prime}(\xi)}{f^{2}(\xi)} \\
v(\xi)=A_{0}+A_{1} f(\xi)+B_{1} \frac{1}{f(\xi)}+A_{2} f^{2}(\xi)+B_{2} \frac{1}{f^{2}(\xi)}+L_{2} f^{\prime}(\xi)+H_{1} \frac{f^{\prime}(\xi)}{f(\xi)}+H_{2} \frac{f^{\prime}(\xi)}{f^{2}(\xi)}
\end{gathered}
$$

where $a_{0}, a_{1}, a_{2}, b_{1}, b_{2}, c_{2}, d_{1}, d_{2}, A_{0}, A_{1}, B_{1}, B_{2}, L_{2}, H_{1}$, and $H_{2}$ are constants to be determined later. Substituting (3.6) with the condition (2.5) into (3.3) and (3.4) and collecting all terms with the same power of $f^{i}(\xi)\left[f^{\prime}(\xi)\right]^{j},(i=\ldots,-2,-1,0,1,2, \ldots ; j=0,1)$. Setting each coefficients of this polynomial to be zero, we get a system of algebraic equations which can be solved by Maple or Mathematica to get the following solutions.

Case 1. Consider

$$
\begin{gathered}
a_{0}=a_{1}=a_{2}=b_{1}=c_{2}=d_{1}=d_{2}=A_{1}=A_{2}=B_{1}=L_{1}=H_{1}=H_{2}=0, \\
A_{0}=\text { arbitrary constant, } \quad b_{2}=-\frac{9 \omega^{2} r}{8 q}, \quad B_{2}=\frac{9 \omega^{3} r}{8 q}, \quad k= \pm \frac{\omega \sqrt{3}}{2 \sqrt{q}} .
\end{gathered}
$$

Case 2. Consider

$$
\begin{gathered}
a_{0}=a_{1}=b_{2}=b_{1}=c_{2}=d_{1}=d_{2}=A_{1}=B_{2}=B_{1}=L_{1}=H_{1}=H_{2}=0, \\
A_{0}=\text { arbitrary constant, } \quad a_{2}=-\frac{9 \omega^{2} p}{8 q}, \quad A_{2}=\frac{9 \omega^{3} p}{8 q}, \quad k= \pm \frac{\omega \sqrt{3}}{2 \sqrt{q}} .
\end{gathered}
$$

Case 3. Consider

$$
\begin{gathered}
a_{0}=a_{1}=a_{2}=b_{1}=c_{2}=d_{1}=A_{2}=A_{1}=B_{1}=L_{1}=H_{1}=0 \\
b_{2}=-\frac{9 \omega^{2} r}{4 q}, \quad d_{2}=\mp \frac{9 \omega^{2} \sqrt{r}}{4 q}, \quad B_{2}=\frac{9 \omega^{3} r}{4 q}, \quad H_{2}= \pm \frac{9 \omega^{3} \sqrt{r}}{4 q} \\
A_{0}=\text { arbitrary constant }, \quad k=\frac{\omega \sqrt{3}}{\sqrt{q}} .
\end{gathered}
$$

Case 4. Consider

$$
\begin{gathered}
a_{0}=a_{1}=b_{1}=c_{2}=d_{1}=d_{2}=A_{1}=B_{1}=L_{1}=H_{1}=H_{2}=0, \\
a_{2}=-\frac{9 \omega^{2} p}{8 q}, \quad b_{2}=-\frac{9 \omega^{2} r}{8 q}, \quad A_{2}=\frac{9 \omega^{3} p}{8 q}, \quad B_{2}=\frac{9 \omega^{3} r}{8 q}, \\
A_{0}=\text { arbitrary constant }, \quad k= \pm \frac{\omega \sqrt{3}}{2 \sqrt{q}} .
\end{gathered}
$$


Note that there are other cases which are omitted here. Since the solutions obtained here are so many, we just list some of the exact solutions corresponding to Case 4 to illustrate the effectiveness of the extended mapping method.

Substituting (3.10) into (3.6) yields

$$
\begin{aligned}
& u(\xi)=-\frac{9 \omega^{2} p}{8 q} f^{2}(\xi)-\frac{9 \omega^{2} r}{8 q} \frac{1}{f^{2}(\xi)} \\
& v(\xi)=\frac{9 \omega^{3} p}{8 q} f^{2}(\xi)+\frac{9 \omega^{3} r}{8 q} \frac{1}{f^{2}(\xi)}
\end{aligned}
$$

where

$$
\xi= \pm \frac{\omega \sqrt{3}}{2 \sqrt{q}}(x-\omega t)
$$

According to Appendix A, we have the following families of exact solutions.

Family 1. If $r=1, q=-\left(1+m^{2}\right), p=m^{2}, f(\xi)=s n(\xi)$, then we get

$$
\begin{gathered}
u(\xi)=\frac{9 \omega^{2}}{8\left(1+m^{2}\right)}\left[m^{2} \mathrm{sn}^{2}(\xi)+\mathrm{ns}^{2}(\xi)\right] \\
v(\xi)=-\frac{9 \omega^{3}}{8\left(1+m^{2}\right)}\left[m^{2} \mathrm{sn}^{2}(\xi)+\mathrm{ns}^{2}(\xi)\right]
\end{gathered}
$$

where

$$
\xi= \pm \frac{\omega \sqrt{3}}{2 i \sqrt{1+m^{2}}}(x-\omega t)
$$

Family 2. If $r=1-m^{2}, q=2 m^{2}-1, p=-m^{2}, f(\xi)=\mathrm{cn}(\xi)$, then we get

$$
\begin{aligned}
& u(\xi)=\frac{9 \omega^{2}}{8\left(2 m^{2}-1\right)}\left[m^{2} \mathrm{cn}^{2}(\xi)-\left(1-m^{2}\right) \mathrm{nc}^{2}(\xi)\right], \\
& v(\xi)=-\frac{9 \omega^{3} m^{2}}{8\left(2 m^{2}-1\right)}\left[\mathrm{cn}^{2}(\xi)-\left(1-m^{2}\right) n c^{2}(\xi)\right],
\end{aligned}
$$

where

$$
\xi= \pm \frac{\omega \sqrt{3}}{2 \sqrt{2 m^{2}-1}}(x-\omega t)
$$


Family 3. If $r=m^{2}-1, q=2-m^{2}, p=-1, f(\xi)=\operatorname{dn}(\xi)$, then we get

$$
\begin{gathered}
u(\xi)=\frac{9 \omega^{2}}{8\left(2-m^{2}\right)}\left[\operatorname{dn}^{2}(\xi)-\left(m^{2}-1\right) \mathrm{nd}^{2}(\xi)\right], \\
v(\xi)=-\frac{9 \omega^{3}}{8\left(2-m^{2}\right)}\left[\operatorname{dn}^{2}(\xi)-\left(m^{2}-1\right) n \mathrm{~d}^{2}(\xi)\right],
\end{gathered}
$$

where

$$
\xi= \pm \frac{\omega \sqrt{3}}{2 \sqrt{m^{2}-1}}(x-\omega t)
$$

Family 4. If $r=m^{2}, q=-\left(1+m^{2}\right), p=1, f(\xi)=\mathrm{dc}(\xi)$, then we get

$$
\begin{gathered}
u(\xi)=\frac{9 \omega^{2}}{8\left(1+m^{2}\right)}\left[\operatorname{dc}^{2}(\xi)+m^{2} \mathrm{~cd}^{2}(\xi)\right], \\
v(\xi)=-\frac{9 \omega^{3}}{8\left(1+m^{2}\right)}\left[\mathrm{dc}^{2}(\xi)+m^{2} \mathrm{~cd}^{2}(\xi)\right],
\end{gathered}
$$

where

$$
\xi= \pm \frac{\omega \sqrt{3}}{2 i \sqrt{1+m^{2}}}(x-\omega t)
$$

Family 5. If $r=1, q=2-m^{2}, p=1-m^{2}, f(\xi)=\mathrm{sc}(\xi)$, then we get

$$
\begin{aligned}
& u(\xi)=-\frac{9 \omega^{2}}{8\left(2-m^{2}\right)}\left[\left(1-m^{2}\right) \operatorname{sc}^{2}(\xi)+\operatorname{cs}^{2}(\xi)\right], \\
& v(\xi)=\frac{9 \omega^{3}}{8\left(2-m^{2}\right)}\left[\left(1-m^{2}\right) \operatorname{sc}^{2}(\xi)+\operatorname{cs}^{2}(\xi)\right],
\end{aligned}
$$

where

$$
\xi= \pm \frac{\omega \sqrt{3}}{2 \sqrt{2-m^{2}}}(x-\omega t)
$$

Family 6. If $r=1 / 4, q=(1 / 2)\left(1-2 m^{2}\right), p=1 / 4, f(\xi)=\mathrm{ns}(\xi) \pm \operatorname{cs}(\xi)$, then we get

$$
\begin{gathered}
u(\xi)=\frac{9 \omega^{2}\left(1-2 \mathrm{~ns}^{2}(\xi)\right)}{8\left(1-2 m^{2}\right)}, \\
v(\xi)=-\frac{9 \omega^{3}\left(1-2 \mathrm{~ns}^{2}(\xi)\right)}{8\left(1-2 m^{2}\right)},
\end{gathered}
$$


where

$$
\xi= \pm \frac{\omega \sqrt{3}}{2 \sqrt{(1 / 2)\left(1-2 m^{2}\right)}}(x-\omega t)
$$

Family 7. If $r=(1 / 4)\left(1-m^{2}\right), q=(1 / 4)\left(1+m^{2}\right), p=(1 / 4)\left(1-m^{2}\right), f(\xi)=\mathrm{nc}(\xi) \pm \mathrm{sc}(\xi)$, then we get

$$
\begin{aligned}
& u(\xi)=-\frac{9 \omega^{2}\left(1-m^{2}\right)\left(\mathrm{sc}^{2}(\xi)+\mathrm{nc}^{2}(\xi)\right)}{4\left(1+m^{2}\right)} \\
& v(\xi)=\frac{9 \omega^{3}\left(1-m^{2}\right)\left(\mathrm{sc}^{2}(\xi)+\mathrm{nc}^{2}(\xi)\right)}{4\left(1+m^{2}\right)}
\end{aligned}
$$

where

$$
\xi= \pm \frac{\omega \sqrt{3}}{\sqrt{1+m^{2}}}(x-\omega t)
$$

Similarly, we can write down the other families of exact solutions of (3.1) which are omitted for convenience.

Example 3.2 (the coupled $\mathrm{KdV}$ equations). In this subsection, consider the coupled $\mathrm{KdV}$ equations [32]:

$$
\begin{aligned}
& u_{t}=u_{x x x}+6 u u_{x}+6 v v_{x}, \\
& v_{t}=v_{x x x}+6 u v_{x}+6 v u_{x} .
\end{aligned}
$$

Substituting (2.2) into (3.27) yields

$$
\begin{gathered}
\omega u^{\prime}+k^{2} u^{\prime \prime \prime}+3\left(u^{2}+v^{2}\right)^{\prime}=0 \\
\omega v^{\prime}+k^{2} v^{\prime \prime \prime}+6(u v)^{\prime}=0 .
\end{gathered}
$$

Integrating (3.2) with respect to $\xi$ once and taking the constant of integration to be zero, we obtain

$$
\begin{gathered}
\omega u+k^{2} u^{\prime \prime}+3\left(u^{2}+v^{2}\right)=0 \\
\omega v+k^{2} v^{\prime \prime}+6(u v)=0 .
\end{gathered}
$$


Suppose that the solutions of (3.27) can be expressed by

$$
\begin{aligned}
& u(\xi)=a_{0}+\sum_{i=1}^{n}\left(a_{i} f^{i}(\xi)+b_{i} f^{-i}(\xi)\right)+\sum_{i=2}^{n} c_{i} f^{i-2}(\xi) f^{\prime}(\xi)+\sum_{i=-1}^{-n} d_{i} f^{i}(\xi) f^{\prime}(\xi) \\
& v(\xi)=\alpha_{0}+\sum_{i=1}^{m}\left(\alpha_{i} f^{i}(\xi)+\beta_{i} f^{-i}(\xi)\right)+\sum_{i=2}^{m} \gamma_{i} f^{i-2}(\xi) f^{\prime}(\xi)+\sum_{i=-1}^{-m} e_{i} f^{i}(\xi) f^{\prime}(\xi)
\end{aligned}
$$

where $a_{0}, a_{i}, b_{i}, c_{i}, d_{i}, \alpha_{i}, \beta_{i}, \gamma_{i}$, and $e_{i}$ are constants to be determined later.

Balancing the order of $u^{\prime \prime}$ and $v^{2}$ in (3.29), the order of $v^{\prime \prime}$ and $u v$ in (3.30), then we can obtain $n=m=2$, so (3.31) can be rewritten as

$$
\begin{aligned}
& u(\xi)=a_{0}+a_{1} f(\xi)+b_{1} \frac{1}{f(\xi)}+a_{2} f^{2}(\xi)+b_{2} \frac{1}{f^{2}(\xi)}+c_{2} f^{\prime}(\xi)+d_{1} \frac{f^{\prime}(\xi)}{f(\xi)}+d_{2} \frac{f^{\prime}(\xi)}{f^{2}(\xi)} \\
& v(\xi)=\alpha_{0}+\alpha_{1} f(\xi)+\beta_{1} \frac{1}{f(\xi)}+\alpha_{2} f^{2}(\xi)+\beta_{2} \frac{1}{f^{2}(\xi)}+\gamma_{2} f^{\prime}(\xi)+e_{1} \frac{f^{\prime}(\xi)}{f(\xi)}+e_{2} \frac{f^{\prime}(\xi)}{f^{2}(\xi)}
\end{aligned}
$$

where $a_{0}, a_{1}, a_{2}, b_{1}, b_{2}, c_{2}, d_{1}, d_{2}, \alpha_{0}, \alpha_{1}, \beta_{1}, \beta_{2}, \gamma_{2}, e_{1}$, and $e_{2}$ are constants to be determined later. Substituting (3.31) with the condition (2.5) into (3.29) and (3.30) and collecting all terms with the same power of $f^{i}(\xi)\left[f^{\prime}(\xi)\right]^{j},(i=\ldots,-2,-1,0,1,2, \ldots ; j=0,1)$. Setting each coefficient of this polynomial to be zero, we get a system of algebraic equations which can be solved by Maple or Mathematica to get the following solutions.

Case 1. Consider

$$
\begin{gathered}
a_{1}=a_{2}=b_{1}=c_{2}=d_{1}=d_{2}=\alpha_{1}=\alpha_{2}=\beta_{1}=e_{1}=e_{2}=\gamma_{2}=0 \\
a_{0}=-\frac{\omega}{12}\left(1+\frac{q}{\sqrt{q^{2}-3 p r}}\right), \quad b_{2}=-\frac{\omega r}{4 \sqrt{q^{2}-3 p r}} \\
\alpha_{0}=-\frac{\omega}{12}\left(1+\frac{q}{\sqrt{q^{2}-3 p r}}\right), \quad \beta_{2}=-\frac{\omega r}{4 \sqrt{q^{2}-3 p r}}, \quad k= \pm \frac{\sqrt{\omega}}{2 \sqrt[4]{q^{2}-3 p r}}
\end{gathered}
$$

Case 2. Consider

$$
\begin{gathered}
a_{1}=b_{1}=b_{2}=c_{2}=d_{1}=d_{2}=\alpha_{1}=\beta_{1}=\beta_{2}=e_{1}=e_{2}=\gamma_{2}=0 \\
a_{0}=-\frac{\omega}{12}\left(1+\frac{q}{\sqrt{q^{2}-3 p r}}\right), \quad a_{2}=-\frac{\omega p}{4 \sqrt{q^{2}-3 p r}}, \\
\alpha_{0}=\frac{\omega}{12}\left(1+\frac{q}{\sqrt{q^{2}-3 p r}}\right), \quad \alpha_{2}=\frac{\omega p}{4 \sqrt{q^{2}-3 p r}}, \quad k= \pm \frac{\sqrt{\omega}}{2 \sqrt[4]{q^{2}-3 p r}} .
\end{gathered}
$$


Case 3. Consider

$$
\begin{gathered}
a_{1}=b_{1}=c_{2}=d_{1}=d_{2}=\alpha_{1}=\beta_{2}=e_{1}=e_{2}=\gamma_{2}=0 \\
a_{0}=-\frac{\omega}{12}\left(3+\frac{q}{\sqrt{q^{2}+12 p r}}\right), \quad a_{2}=-\frac{\omega p}{4 \sqrt{q^{2}+12 p r}}, \quad b_{2}=-\frac{\omega r}{4 \sqrt{q^{2}+12 p r}}, \\
\alpha_{0}=-\frac{\omega}{12}\left(1-\frac{q}{\sqrt{q^{2}+12 p r}}\right), \quad \alpha_{2}=\frac{\omega p}{4 \sqrt{q^{2}+12 p r}}, \quad \beta_{2}=\frac{\omega r}{4 \sqrt{q^{2}+12 p r}} \\
k= \pm \frac{\sqrt{\omega}}{2 \sqrt[4]{q^{2}+12 p r}} .
\end{gathered}
$$

Case 4. Consider

$$
\begin{gathered}
a_{1}=b_{1}=b_{2}=d_{1}=d_{2}=\alpha_{1}=\beta_{1}=\beta_{2}=e_{1}=e_{2}=0, \\
a_{0}=-\frac{\omega}{12}\left(1+\frac{q}{\sqrt{q^{2}+12 p r}}\right), \quad a_{2}=-\frac{\omega p}{2 \sqrt{q^{2}+12 p r}}, \quad c_{2}=-\frac{\omega \sqrt{p}}{2 \sqrt{q^{2}+12 p r}}, \\
\alpha_{0}=\frac{\omega}{12}\left(1+\frac{q}{\sqrt{q^{2}+12 p r}}\right), \quad \alpha_{2}=\frac{\omega p}{2 \sqrt{q^{2}+12 p r}}, \quad \gamma_{2}=\frac{\omega \sqrt{p}}{2 \sqrt{q^{2}+12 p r}}, \\
k= \pm \frac{\sqrt{\omega}}{\sqrt[4]{q^{2}+12 p r}} .
\end{gathered}
$$

Note that there are other cases which are omitted here. Since the solutions obtained here are so many, we just list some of the exact solutions corresponding to Case 4 to illustrate the effectiveness of the extended mapping method.

Substituting (3.36) into (3.32) yields

$$
\begin{aligned}
& u(\xi)=-\frac{\omega}{12}\left(1+\frac{q}{\sqrt{q^{2}+12 p r}}\right)-\frac{\omega p}{2 \sqrt{q^{2}+12 p r}} f^{2}(\xi)-\frac{\omega \sqrt{p}}{2 \sqrt{q^{2}+12 p r}} f^{\prime}(\xi), \\
& v(\xi)=\frac{\omega}{12}\left(1+\frac{q}{\sqrt{q^{2}+12 p r}}\right)+\frac{\omega p}{2 \sqrt{q^{2}+12 p r}} f^{2}(\xi)+\frac{\omega \sqrt{p}}{2 \sqrt{q^{2}+12 p r}} f^{\prime}(\xi),
\end{aligned}
$$


where

$$
\xi= \pm \frac{\sqrt{\omega}}{\sqrt[4]{q^{2}+12 p r}}(x-\omega t) .
$$

According to Appendix A, we have the following families of exact solutions.

Family 1. If $r=1, q=2 m^{2}-1, p=m^{2}\left(m^{2}-1\right), f(\xi)=\operatorname{sd}(\xi)$, then we get

$$
\begin{aligned}
& u(\xi)=-\frac{\omega}{12}\left(1+\frac{2 m^{2}-1}{\sqrt{16 m^{4}-16 m^{2}+1}}\right)-\frac{\omega m^{2}\left(m^{2}-1\right) \mathrm{sd}^{2}(\xi)}{2 \sqrt{16 m^{4}-16 m^{2}+1}}-\frac{\omega m \sqrt{m^{2}-1} \mathrm{nd}(\xi) \mathrm{cd}(\xi)}{2 \sqrt{16 m^{4}-16 m^{2}+1}} \\
& v(\xi)=\frac{\omega}{12}\left(1+\frac{2 m^{2}-1}{\sqrt{16 m^{4}-16 m^{2}+1}}\right)+\frac{\omega m^{2}\left(m^{2}-1\right) \mathrm{sd}^{2}(\xi)}{2 \sqrt{16 m^{4}-16 m^{2}+1}}+\frac{\omega m \sqrt{m^{2}-1} \mathrm{nd}(\xi) \mathrm{cd}(\xi)}{2 \sqrt{16 m^{4}-16 m^{2}+1}}
\end{aligned}
$$

where

$$
\xi= \pm \frac{\sqrt{\omega}}{\sqrt[4]{16 m^{4}-16 m^{2}+1}}(x-\omega t)
$$

Family 2. If $r=m^{2}\left(m^{2}-1\right), q=2 m^{2}-1, p=1, f(\xi)=d s(\xi)$, then we get

$$
\begin{aligned}
& u(\xi)=-\frac{\omega}{12}\left(1+\frac{2 m^{2}-1}{\sqrt{16 m^{4}-16 m^{2}+1}}\right)-\frac{\omega \mathrm{ds}^{2}(\xi)}{2 \sqrt{16 m^{4}-16 m^{2}+1}}+\frac{\omega \operatorname{cs}(\xi) \mathrm{ns}(\xi)}{2 \sqrt{16 m^{4}-16 m^{2}+1}}, \\
& v(\xi)=\frac{\omega}{12}\left(1+\frac{2 m^{2}-1}{\sqrt{16 m^{4}-16 m^{2}+1}}\right)+\frac{\omega \mathrm{ds}^{2}(\xi)}{2 \sqrt{16 m^{4}-16 m^{2}+1}}-\frac{\omega \operatorname{cs}(\xi) \mathrm{ns}(\xi)}{2 \sqrt{16 m^{4}-16 m^{2}+1}},
\end{aligned}
$$

where

$$
\xi= \pm \frac{\sqrt{\omega}}{\sqrt[4]{16 m^{4}-16 m^{2}+1}}(x-\omega t)
$$

Family 3. If $r=m^{2} / 4, q=(1 / 2)\left(m^{2}-2\right), p=m^{2} / 4, f(\xi)=\operatorname{sn}(\xi) \pm i \mathrm{cn}(\xi)$, then we get

$$
\begin{aligned}
& u(\xi)=-\frac{\omega}{12}\left(1+\frac{m^{2}-2}{2 \sqrt{m^{4}-m^{2}+1}}\right)-\frac{\omega m^{2}(\operatorname{sn}(\xi) \pm i \operatorname{cn}(\xi))^{2}}{8 \sqrt{m^{4}-m^{2}+1}}-\frac{\omega m(c n(\xi) \operatorname{dn}(\xi) \mp i \operatorname{sn}(\xi) \operatorname{dn}(\xi))}{4 \sqrt{m^{4}-m^{2}+1}} \\
& v(\xi)=\frac{\omega}{12}\left(1+\frac{m^{2}-2}{2 \sqrt{m^{4}-m^{2}+1}}\right)+\frac{\omega m^{2}(\operatorname{sn}(\xi) \pm i c n(\xi))^{2}}{8 \sqrt{m^{4}-m^{2}+1}}+\frac{\omega m(\operatorname{cn}(\xi) \operatorname{dn}(\xi) \mp i \operatorname{sn}(\xi) \operatorname{dn}(\xi))}{4 \sqrt{m^{4}-m^{2}+1}}
\end{aligned}
$$


where

$$
\xi= \pm \frac{\sqrt{\omega}}{\sqrt[4]{m^{4}-m^{2}+1}}(x-\omega t)
$$

Family 4. If $r=1, q=-\left(1+m^{2}\right), p=m^{2}, f(\xi)=\operatorname{sn}(\xi)$, then we get

$$
\begin{aligned}
& u(\xi)=-\frac{\omega}{12}\left(1-\frac{1+m^{2}}{\sqrt{m^{4}+14 m^{2}+1}}\right)-\frac{\omega m^{2} \operatorname{sn}^{2}(\xi)}{2 \sqrt{m^{4}+14 m^{2}+1}}-\frac{\omega m \mathrm{cn}(\xi) \mathrm{dn}(\xi)}{2 \sqrt{m^{4}+14 m^{2}+1}} \\
& v(\xi)=\frac{\omega}{12}\left(1-\frac{1+m^{2}}{\sqrt{m^{4}+14 m^{2}+1}}\right)+\frac{\omega m^{2} \operatorname{sn}^{2}(\xi)}{2 \sqrt{m^{4}+14 m^{2}+1}}+\frac{\omega m \mathrm{cn}(\xi) \mathrm{dn}(\xi)}{2 \sqrt{m^{4}+14 m^{2}+1}}
\end{aligned}
$$

where

$$
\xi= \pm \frac{\sqrt{\omega}}{\sqrt[4]{m^{4}+14 m^{2}+1}}(x-\omega t)
$$

Family 5. If $r=1-m^{2}, q=2 m^{2}-1, p=-m^{2}, f(\xi)=\mathrm{cn}(\xi)$, then we get

$$
\begin{aligned}
& u(\xi)=-\frac{\omega}{12}\left(1+\frac{2 m^{2}-1}{\sqrt{16 m^{4}-16 m^{2}+1}}\right)+\frac{\omega m^{2} \mathrm{cn}^{2}(\xi)}{2 \sqrt{16 m^{4}-16 m^{2}+1}}+\frac{i \omega m \operatorname{sn}(\xi) \mathrm{dn}(\xi)}{2 \sqrt{16 m^{4}-16 m^{2}+1}}, \\
& v(\xi)=\frac{\omega}{12}\left(1+\frac{2 m^{2}-1}{\sqrt{16 m^{4}-16 m^{2}+1}}\right)-\frac{\omega m^{2} \mathrm{cn}^{2}(\xi)}{2 \sqrt{16 m^{4}-16 m^{2}+1}}-\frac{i \omega m \operatorname{sn}(\xi) \mathrm{dn}(\xi)}{2 \sqrt{16 m^{4}-16 m^{2}+1}},
\end{aligned}
$$

where

$$
\xi= \pm \frac{\sqrt{\omega}}{\sqrt[4]{16 m^{4}-16 m^{2}+1}}(x-\omega t)
$$

Family 6. If $r=1-m^{2}, q=2-m^{2}, p=1, f(\xi)=\operatorname{cs}(\xi)$, then we get

$$
\begin{aligned}
& u(\xi)=-\frac{\omega}{12}\left(1+\frac{2-m^{2}}{\sqrt{m^{4}-16 m^{2}+16}}\right)-\frac{\omega \operatorname{cs}^{2}(\xi)}{2 \sqrt{m^{4}-16 m^{2}+16}}+\frac{\omega n s(\xi) \mathrm{ds}(\xi)}{2 \sqrt{m^{4}-16 m^{2}+16}} \\
& v(\xi)=\frac{\omega}{12}\left(1+\frac{2-m^{2}}{\sqrt{m^{4}-16 m^{2}+16}}\right)+\frac{\omega \operatorname{cs}^{2}(\xi)}{2 \sqrt{m^{4}-16 m^{2}+16}}-\frac{\omega \operatorname{ns}(\xi) \mathrm{ds}(\xi)}{2 \sqrt{m^{4}-16 m^{2}+16}}
\end{aligned}
$$

where

$$
\xi= \pm \frac{\sqrt{\omega}}{\sqrt[4]{m^{4}-16 m^{2}+16}}(x-\omega t)
$$


Table 1

\begin{tabular}{|c|c|c|c|c|}
\hline$p$ & $q$ & $r$ & $f(\xi)$ & $f^{\prime}(\xi)$ \\
\hline$m^{2}$ & $-\left(1+m^{2}\right)$ & 1 & $\mathrm{sn}(\xi)$ & $\mathrm{cn}(\xi) \mathrm{dn}(\xi)$ \\
\hline$-m^{2}$ & $2 m^{2}-1$ & $1-m^{2}$ & $\operatorname{cn}(\xi)$ & $-\operatorname{sn}(\xi) d n(\xi)$ \\
\hline-1 & $2-m^{2}$ & $m^{2}-1$ & $\operatorname{dn}(\xi)$ & $-m^{2} \operatorname{sn}(\xi) \operatorname{cn}(\xi)$ \\
\hline 1 & $-\left(1+m^{2}\right)$ & $m^{2}$ & $\mathrm{~ns}(\xi)$ & $-\mathrm{ds}(\xi) \operatorname{cs}(\xi)$ \\
\hline$m^{2}-1$ & $2-m^{2}$ & -1 & $\operatorname{nd}(\xi)$ & $m^{2} \operatorname{sd}(\xi) \operatorname{cd}(\xi)$ \\
\hline 1 & $2-m^{2}$ & $1-m^{2}$ & $\operatorname{cs}(\xi)$ & $-\mathrm{ns}(\xi) \mathrm{ds}(\xi)$ \\
\hline $1-m^{2}$ & $2-m^{2}$ & 1 & $\mathrm{sc}(\xi)$ & $\mathrm{nc}(\xi) \mathrm{dc}(\xi)$ \\
\hline$m^{2}\left(m^{2}-1\right)$ & $2 m^{2}-1$ & 1 & $\operatorname{sd}(\xi)$ & $\operatorname{nd}(\xi) \operatorname{cd}(\xi)$ \\
\hline 1 & $2 m^{2}-1$ & $m^{2}\left(m^{2}-1\right)$ & $\mathrm{ds}(\xi)$ & $-\mathrm{cs}(\xi) \mathrm{ns}(\xi)$ \\
\hline$\frac{1}{4}$ & $\frac{1}{2}\left(1-2 m^{2}\right)$ & $\frac{1}{4}$ & $\operatorname{ns}(\xi) \pm \operatorname{cs}(\xi)$ & $-\mathrm{ds}(\xi) \operatorname{cs}(\xi) \mp \mathrm{ns}(\xi) \mathrm{ds}(\xi)$ \\
\hline$\frac{1}{4}\left(1-m^{2}\right)$ & $\frac{1}{4}\left(1+m^{2}\right)$ & $\frac{1}{4}\left(1-m^{2}\right)$ & $\mathrm{nc}(\xi) \pm \mathrm{sc}(\xi)$ & $\mathrm{sc}(\xi) \mathrm{dc}(\xi) \pm \mathrm{nc}(\xi) \mathrm{dc}(\xi)$ \\
\hline$\frac{m^{2}}{4}$ & $\frac{1}{2}\left(m^{2}-2\right)$ & $\frac{m^{2}}{4}$ & $\operatorname{sn}(\xi) \pm i \operatorname{cn}(\xi)$ & $\operatorname{cn}(\xi) \operatorname{dn}(\xi) \mp i \operatorname{sn}(\xi) \operatorname{dn}(\xi)$ \\
\hline
\end{tabular}

Table 2

\begin{tabular}{llll}
\hline $\operatorname{sn}(\xi) \rightarrow \tanh (\xi)$ & $\operatorname{cn}(\xi) \rightarrow \operatorname{sech}(\xi)$ & $\operatorname{dn}(\xi) \rightarrow \operatorname{sech}(\xi)$ & $\operatorname{ns}(\xi) \rightarrow \operatorname{coth}(\xi)$ \\
$\operatorname{cs}(\xi) \rightarrow \operatorname{csch}(\xi)$ & $\mathrm{ds}(\xi) \rightarrow \operatorname{csch}(\xi)$ & $\operatorname{sc}(\xi) \rightarrow \sinh (\xi)$ & $\operatorname{sd}(\xi) \rightarrow \sinh (\xi)$ \\
\hline
\end{tabular}

Family 7. If $r=-1, q=2-m^{2}, p=m^{2}-1, f(\xi)=\mathrm{nd}(\xi)$, then we get

$$
\begin{aligned}
& u(\xi)=-\frac{\omega}{12}\left(1+\frac{2-m^{2}}{\sqrt{m^{4}-16 m^{2}+16}}\right)-\frac{\omega\left(m^{2}-1\right) \mathrm{nd}^{2}(\xi)}{2 \sqrt{m^{4}-16 m^{2}+16}}-\frac{\omega m^{2} \sqrt{m^{2}-1} \mathrm{sd}(\xi) \mathrm{cd}(\xi)}{2 \sqrt{m^{4}-16 m^{2}+16}} \\
& v(\xi)=\frac{\omega}{12}\left(1+\frac{2-m^{2}}{\sqrt{m^{4}-16 m^{2}+16}}\right)+\frac{\omega\left(m^{2}-1\right) \mathrm{nd}^{2}(\xi)}{2 \sqrt{m^{4}-16 m^{2}+16}}+\frac{\omega m^{2} \sqrt{m^{2}-1} \mathrm{sd}(\xi) \mathrm{cd}(\xi)}{2 \sqrt{m^{4}-16 m^{2}+16}}
\end{aligned}
$$

where

$$
\xi= \pm \frac{\sqrt{\omega}}{\sqrt[4]{m^{4}-16 m^{2}+16}}(x-\omega t)
$$

\section{Conclusion}

The main objective of this paper is that we have found new exact solutions for the Boussinesq system and the coupled $\mathrm{KdV}$ equations by using the extended mapping method with the auxiliary equation method. Also, we conclude according to Appendix B that our results in terms of Jacobi elliptic functions generate into hyperbolic functions when $m \rightarrow 1$ and generate into trigonometric functions when $m \rightarrow 0$. This method provides a powerful mathematical tool to obtain more general exact solutions of a great many nonlinear PDEs in mathematical physics. 
Table 3

\begin{tabular}{llll}
\hline $\mathrm{nc}(\xi)=\frac{1}{\operatorname{cn}(\xi)}$ & $\operatorname{nd}(\xi)=\frac{1}{\operatorname{dn}(\xi)}$ & $\operatorname{cd}(\xi)=\frac{\operatorname{cn}(\xi)}{\operatorname{dn}(\xi)}$ & $\operatorname{dc}(\xi)=\frac{\operatorname{dn}(\xi)}{\operatorname{cn}(\xi)}$ \\
$\operatorname{cs}(\xi)=\frac{\operatorname{cn}(\xi)}{\operatorname{sn}(\xi)}$ & $\operatorname{sc}(\xi)=\frac{\operatorname{sn}(\xi)}{\operatorname{cn}(\xi)}$ & $\operatorname{sd}(\xi)=\frac{\operatorname{sn}(\xi)}{\operatorname{dn}(\xi)}$ & $\operatorname{ds}(\xi)=\frac{\operatorname{dn}(\xi)}{\operatorname{sn}(\xi)}$ \\
\hline
\end{tabular}

\section{Appendices}

\section{A. The Jacobi Elliptic Functions}

The general solutions to the Jacobi elliptic equation (2.3) and its derivatives [31] are listed in Table 1 , where $0<m<1$ is the modulus of the Jacobi elliptic functions and $i=\sqrt{-1}$.

\section{B. Hyperbolic Functions}

The Jacobi elliptic functions $\operatorname{sn}(\xi), \operatorname{cn}(\xi), \operatorname{dn}(\xi), \operatorname{ns}(\xi), \operatorname{cs}(\xi), \mathrm{ds}(\xi), \operatorname{sc}(\xi), \operatorname{sd}(\xi)$ generate into hyperbolic functions when $m \rightarrow 1$ as in Table 2 .

\section{Relations between the Jacobi Elliptic Functions}

See Table 3.

\section{References}

[1] M. J. Ablowitz and P. A. Clarkson, Soliton, Nonlinear Evolution Equations and Inverse Scattering, Cambridge University Press, Cambridge, UK, 1991.

[2] C. H. Gu, H. S. Hu, and Z. X. Zhou, Soliton Theory and Its Application, Zhejiang Science and Technology Press, Zhejiang, China, 1990.

[3] V. B. Matveev and M. A. Salle, Darboux transformations and solitons, Springer-Verlag, Berlin, Germany, 1991.

[4] R. Hirota, The Direct Method in Soliton Theory, Cambridge University Press, Cambridge, UK, 2004.

[5] S.-Y. Lou and J. Z. Lu, "Special solutions from the variable separation approach: the Davey-Stewartson equation," Journal of Physics A, vol. 29, no. 14, pp. 4209-4215, 1996.

[6] E. J. Parkes and B. R. Duffy, "Travelling solitary wave solutions to a compound KdV-Burgers equation," Physics Letters A, vol. 229, no. 4, pp. 217-220, 1997.

[7] E. Fan, "Extended tanh-function method and its applications to nonlinear equations," Physics Letters. A, vol. 277 , no. $4-5$, pp. $212-218,2000$.

[8] Z. Y. Yan, "New explicit travelling wave solutions for two new integrable coupled nonlinear evolution equations," Physics Letters A, vol. 292, no. 1-2, pp. 100-106, 2001.

[9] Y. Chen and Z. Yu, "Generalized extended tanh-function method to construct new explicit exact solutions for the approximate equations for long water waves," International Journal of Modern Physics C, vol. 14, no. 5, pp. 601-611, 2003.

[10] M. Wang, Y. Zhou, and Z. Li, "Application of a homogeneous balance method to exact solutions of nonlinear equations in mathematical physics," Physics Letters A, vol. 216, no. 1-5, pp. 67-75, 1996.

[11] G. W. Bluman and S. Kumei, Symmetries and Differential Equations, Springer-Verlag, New York, NY, USA, 1989.

[12] P. J. Olver, Applications of Lie Groups to Differential Equations, Springer-Verlag, New York, NY, USA, 1986.

[13] E. M. E. Zayed and K. A. Gepreel, "The $\left(G^{\prime} / G\right)$-expansion method for finding traveling wave solutions of nonlinear partial differential equations in mathematical physics," Journal of Mathematical Physics, vol. 50, no. 1, p. 12, 2009. 
[14] Z. Y. Yan, "A reduction mKdV method with symbolic computation to construct new doubly-periodic solutions for nonlinear wave equations," International Journal of Modern Physics C, vol. 14, no. 5, pp. 661-672, 2003.

[15] Z. Y. Yan, "The new tri-function method to multiple exact solutions of nonlinear wave equations," Physica Scripta, vol. 78, no. 3, Article ID 035001, p. 5, 2008.

[16] Z. Y. Yan, "Periodic, solitary and rational wave solutions of the 3D extended quantum ZakharovKuznetsov equation in dense quantum plasmas," Physics Letters A, vol. 373, no. 29, pp. 2432-2437, 2009.

[17] D. C. Lu and B. J. Hong, "New exact solutions for the (2+1)-dimensional generalized Broer-Kaup system," Applied Mathematics and Computation, vol. 199, no. 2, pp. 572-580, 2008.

[18] A. V. Porubov, "Periodical solution to the nonlinear dissipative equation for surface waves in a convecting liquid layer," Physics Letters A, vol. 221, no. 6, pp. 391-394, 1996.

[19] A.-M. Wazwaz, "The tanh and the sine-cosine methods for compact and noncompact solutions of the nonlinear Klein-Gordon equation," Applied Mathematics and Computation, vol. 167, no. 2, pp. 11791195, 2005.

[20] Z. Y. Yan and H. Q. Zhang, "New explicit solitary wave solutions and periodic wave solutions for Whitham-Broer-Kaup equation in shallow water," Physics Letters A, vol. 285, no. 5-6, pp. 355-362, 2001.

[21] D. Lü, "Jacobi elliptic function solutions for two variant Boussinesq equations," Chaos, Solitons and Fractals, vol. 24, no. 5, pp. 1373-1385, 2005.

[22] Z. Y. Yan, "Abundant families of Jacobi elliptic function solutions of the (2+1)-dimensional integrable Davey-Stewartson-type equation via a new method," Chaos, Solitons and Fractals, vol. 18, no. 2, pp. 299-309, 2003.

[23] C. L. Bai and H. Zhao, "Generalized method to construct the solitonic solutions to (3+1)-dimensional nonlinear equation," Physics Letters A, vol. 354, no. 5-6, pp. 428-436, 2006.

[24] F. Cariello and M. Tabor, "Similarity reductions from extended Painlevé expansions for nonintegrable evolution equations," Physica D, vol. 53, no. 1, pp. 59-70, 1991.

[25] M. Wang and X. Li, "Extended F-expansion method and periodic wave solutions for the generalized Zakharov equations," Physics Letters A, vol. 343, no. 1-3, pp. 48-54, 2005.

[26] X. Feng, "Exploratory approach to explicit solution of nonlinear evolution equations," International Journal of Theoretical Physics, vol. 39, no. 1, pp. 207-222, 2000.

[27] J. L. Hu, "Explicit solutions to three nonlinear physical models," Physics Letters A, vol. 287, no. 1-2, pp. 81-89, 2001.

[28] J. L. Hu and H. Zhang, "A new method for finding exact traveling wave solutions to nonlinear partial differential equations," Physics Letters A, vol. 286, no. 2-3, pp. 175-179, 2001.

[29] J. H. He and X. H. Wu, "Exp-function method for nonlinear wave equations," Chaos, Solitons and Fractals, vol. 30, no. 3, pp. 700-708, 2006.

[30] X. Li and M. L. Wang, "A sub-ODE method for finding exact solutions of a generalized KdV-mKdV equation with high-order nonlinear terms," Physics Letters A, vol. 361, no. 1-2, pp. 115-118, 2007.

[31] K. A. Gepreel, "Exact solutions for nonlinear PDEs with the variable coefficients in mathematical physics," Journal of Computational Science, vol. 6, no. 1, pp. 003-014, 2011.

[32] J. P. Wang, "A list of 1+1 dimensional integrable equations and their properties," Journal of Nonlinear Mathematical Physics, vol. 9, supplement 1, p. 213, 2002. 


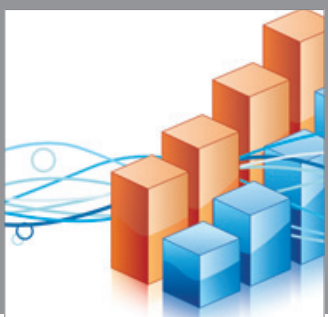

Advances in

Operations Research

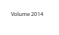

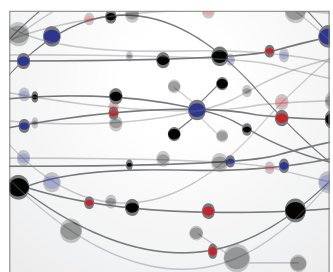

\section{The Scientific} World Journal
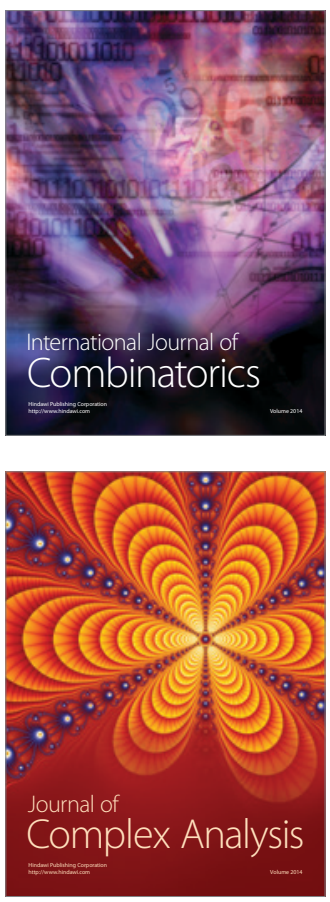

International Journal of

Mathematics and

Mathematical

Sciences
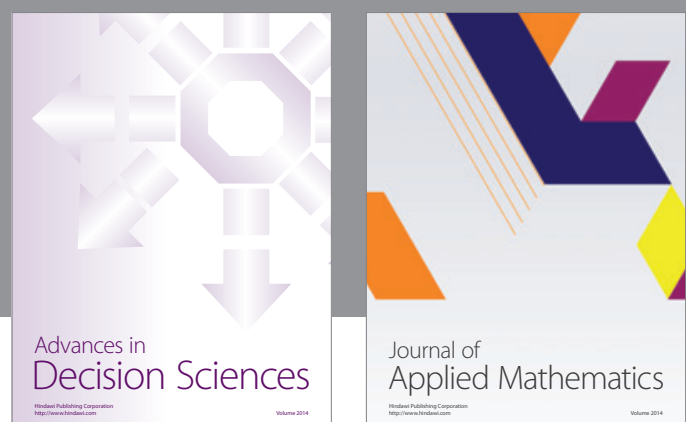

Journal of

Applied Mathematics
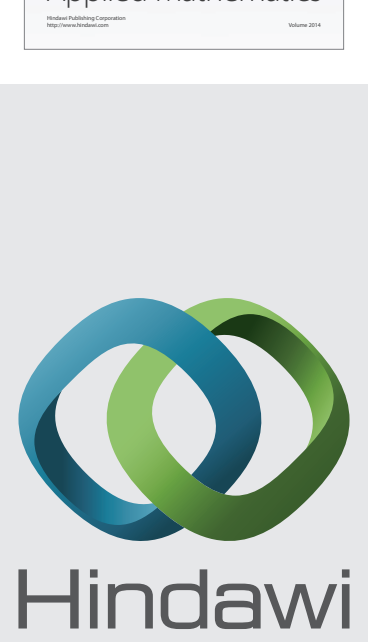

Submit your manuscripts at http://www.hindawi.com
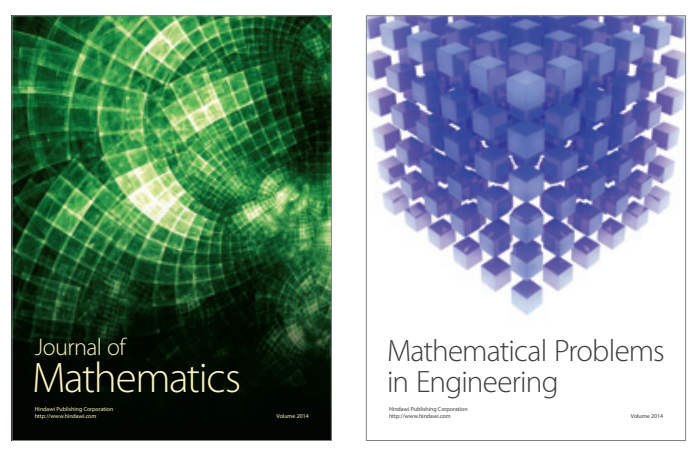

Mathematical Problems in Engineering
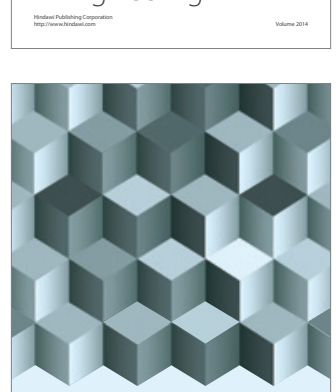

Journal of

Function Spaces
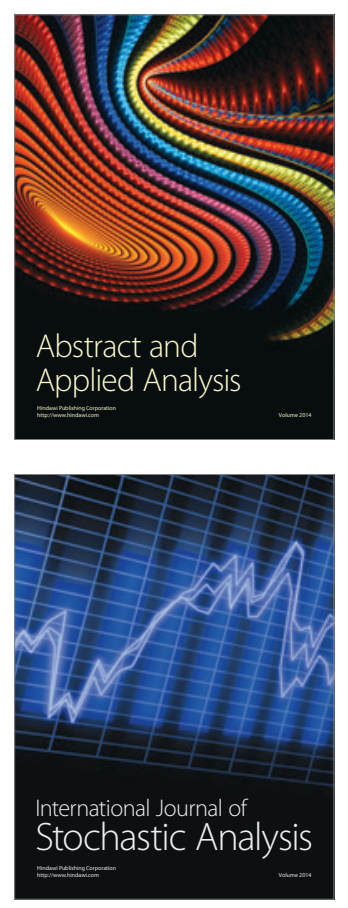

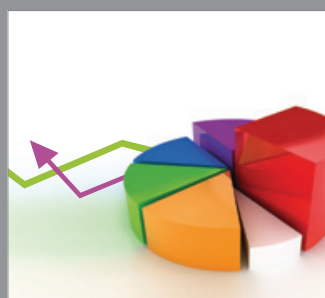

ournal of

Probability and Statistics

Promensencen
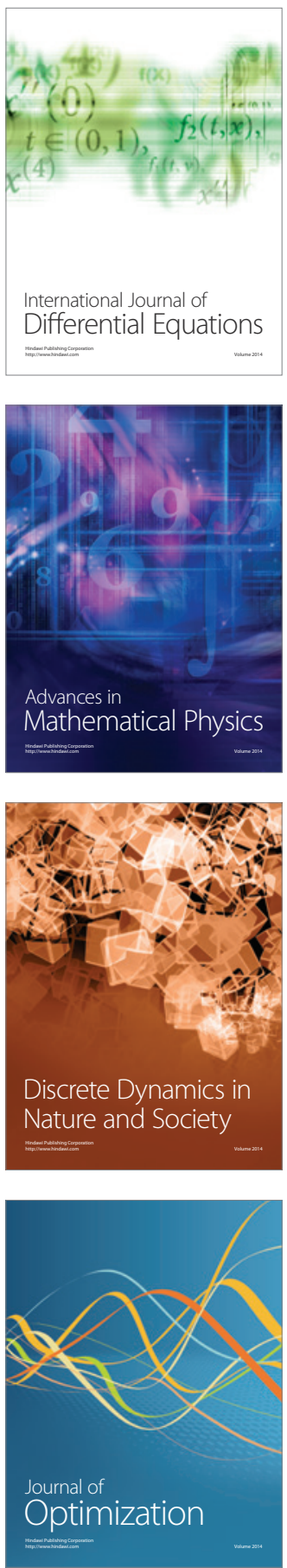